\title{
Vacuum squeezing in atomic media via self-rotation
}

\author{
A. B. Matsko, I. Novikova, and G. R. Welch \\ Department of Physics and Institute for Quantum Studies, Texas A\&M University, College Station, Texas 77843-4242 \\ D. Budker, D. F. Kimball, and S. M. Rochester \\ Department of Physics, University of California, Berkeley, California 94720-7300 \\ (Received 14 November 2001; revised manuscript received 16 July 2002; published 30 October 2002)
}

\begin{abstract}
When linearly polarized light propagates through a medium in which elliptically polarized light would undergo self-rotation, squeezed vacuum can appear in the orthogonal polarization. A simple relationship between self-rotation and the degree of vacuum squeezing is developed. Taking into account absorption, we find the optimum conditions for squeezing in any medium that can produce self-rotation. We then find analytic expressions for the amount of vacuum squeezing produced by an atomic vapor when light is near-resonant with a transition between various low-angular-momentum states. Finally, we consider a gas of multilevel Rb atoms, and analyze squeezing for light tuned near the $D$ lines under realistic conditions.
\end{abstract}

DOI: 10.1103/PhysRevA.66.043815

PACS number(s): 42.50.Dv, 42.50.Gy, 42.50.Ar, 32.60.+i

\section{INTRODUCTION}

Quantum fluctuations are the main factor limiting the precision of many measurements and the signal-to-noise ratio in optical communication. However, shot noise is not a fundamental restriction on the precision of an optical measurement if nonclassical states of light are used [1-6]. For example, squeezed vacuum has been used to improve the performance of optical interferometers beyond the shot-noise limit $[7,8]$. A number of techniques for producing squeezed states of light using nonlinear optics have been developed (see, e.g., Ref. [9] and references therein).

It has been known for some time that if linearly polarized light propagates through a medium that can produce nonlinear self-rotation (SR) of elliptically polarized light (see, e.g., Refs. [10-14] and references therein), the light will be in a squeezed state after traversal of the medium if losses in the medium are sufficiently small. A detailed theoretical study of this effect for a generic Kerr medium without absorption was carried out in Ref. [15]. It can be difficult to achieve efficient squeezing in a realistic Kerr medium because of the absorption and scattering of light. Nonetheless, squeezed electromagnetic vacuum was recently produced in a nonbirefringent optical fiber in which elliptically polarized light undergoes SR [16].

In this paper, we derive a simple formula for the amount of squeezing generated by a medium which exhibits selfrotation, including the effects of absorption (which acts to diminish the squeezing). In atomic vapors, one can take advantage of resonant enhancement of self-rotation (as well as coherence and interference effects [17-25]) to create a medium in which there is large SR and small absorption. Employing density-matrix calculations, we analyze vacuum squeezing of resonant light in some low-angular-momentum atomic systems and in atomic rubidium. We find that under readily achievable experimental conditions, vacuum squeezing of about $8 \mathrm{~dB}$ can be expected.

\section{RELATIONSHIP BETWEEN SELF-ROTATION AND VACUUM SQUEEZING}

Before embarking on the calculations, we present a qualitative explanation of why self-rotation leads to squeezing. Suppose that we have a strong linearly polarized (along $\hat{\mathbf{y}}$ ) light field propagating in the $\hat{\mathbf{z}}$ direction through a medium in which elliptically polarized light would undergo SR. Also suppose there is a weak co-propagating $x$-polarized light field. In general, the resultant field is elliptically polarized and the principal axis of the polarization ellipse will rotate upon propagation through the medium. This rotation projects a portion of the strong field along $\hat{\mathbf{x}}$. Depending on the relative phase between the orthogonally polarized strong and weak input fields, the $x$-polarized output field can be amplified or attenuated compared to the weak input field (phasesensitive gain or loss). In the latter case, we have a negative feedback mechanism which reduces the field along $\hat{\mathbf{x}}$. This analysis applies even when the weak input field is solely due to vacuum fluctuations.

To describe squeezing that accompanies propagation of light through a nonlinear SR medium, we first introduce a phenomenological description of SR. The interaction of light of ellipticity $\epsilon$ with an atomic medium of length $\ell$ can induce circular birefringence and linear dichroism in the medium. This results in rotation by an angle $\varphi$ of the principal axis of the light polarization ellipse. Mechanisms for SR have been discussed in, e.g., Ref. [11]. For optically thin media and small initial ellipticity $\epsilon(0) \ll 1$, the principal axis of the polarization ellipse rotates by an angle

$$
\varphi=g \epsilon(0) \ell,
$$

where we have defined an SR parameter $g$ that, for a given atomic medium, depends only on the incident light intensity and frequency. Because the initial ellipticity of the light field is small, $\varphi \ll 1$. We also assume that $\epsilon$ does not change significantly as light propagates through the medium [i.e., $\epsilon$ is 
independent of $z$, so that $\epsilon(0)=\epsilon(\ell)=\epsilon]$, an assumption that we will later show is justified in concrete examples considered in Secs. IV and VI.

The absorption of light by the medium is characterized by the parameter $\alpha$; the light intensity $I(z)$ as a function of the distance $z$ the light has propagated through the medium is given by $I(z)=I(0) \exp (-\alpha z)$.

The parameters $g$ and $\alpha$ can be measured or calculated (see, Refs. [11-14] and Secs. III and IV); knowledge of these two parameters allows one to calculate the amount of vacuum squeezing produced by a given system, in the following manner. Consider a monochromatic light field $\mathbf{E}(z, t)$ propagating in the $\hat{\mathbf{z}}$ direction represented by

$$
\begin{gathered}
\mathbf{E}(z, t)=E_{x}(z, t) \hat{\mathbf{x}}+E_{y}(z, t) \hat{\mathbf{y}}, \\
E_{x}(z, t)=\mathcal{E}_{x}(z) \cos [k z-\omega t+\phi(z)], \\
E_{y}(z, t)=\mathcal{E}_{y}(z) \cos (k z-\omega t),
\end{gathered}
$$

where $\mathcal{E}_{x}(z)$ and $\mathcal{E}_{y}(z)$ are the real positive amplitudes of the $x$ - and $y$-polarized components of the electromagnetic field, $\omega$ is the light frequency, $k=\omega / c$ is the vacuum wave number, and $\phi(z)$ is the relative phase between the two components. The light field can also be written in terms of its positiveand negative-frequency components, $E_{x, y}^{+}(z, t)$ and $E_{x, y}^{-}(z, t)$, where

$$
\begin{gathered}
\mathbf{E}^{+}(z, t)=\frac{\mathcal{E}_{x}(z)}{2} e^{i[k z-\omega t+\phi(z)]} \hat{\mathbf{x}}+\frac{\mathcal{E}_{y}(z)}{2} e^{i(k z-\omega t)} \hat{\mathbf{y}} \\
\mathbf{E}^{-}(z, t)=\frac{\mathcal{E}_{x}(z)}{2} e^{-i[k z-\omega t+\phi(z)]} \hat{\mathbf{x}}+\frac{\mathcal{E}_{y}(z)}{2} e^{-i(k z-\omega t)} \hat{\mathbf{y}} .
\end{gathered}
$$

The ellipticity $\epsilon$ of the light field is given by (see, e.g., Ref. [29])

$$
\epsilon=\frac{1}{2} \arcsin \frac{i\left(E_{x}^{-} E_{y}^{+}-E_{y}^{-} E_{x}^{+}\right)}{\left|E_{x}\right|^{2}+\left|E_{y}\right|^{2}} .
$$

Assuming that $\mathcal{E}_{y}(z) \gg \mathcal{E}_{x}(z)$, we have for the ellipticity $\epsilon(z)$

$$
\epsilon(z) \approx \frac{\mathcal{E}_{x}(z)}{\mathcal{E}_{y}(z)} \sin \phi(z) .
$$

First we consider an SR medium of length $\ell$ for which absorption is negligible ( $\alpha \ell \approx 0$ ), and relate the optical fields at the output to the input fields, using Eq. (1). For simplicity, we deal only with the positive-frequency components of the optical electric field. The output fields $E_{x}^{+}(\ell)$ and $E_{y}^{+}(\ell)$ are then given by

$$
\left[\begin{array}{c}
E_{x}^{+}(\ell) \\
E_{y}^{+}(\ell)
\end{array}\right] \approx\left[\begin{array}{cc}
1 & \varphi \\
-\varphi & 1
\end{array}\right]\left[\begin{array}{c}
\mathcal{E}_{x}(0) e^{i \phi(0)} \\
\mathcal{E}_{y}(0)
\end{array}\right] e^{i(k \ell-\omega t)}
$$

The positive-frequency part of the $x$-polarized component of the output field is given by

$$
E_{x}^{+}(\ell) \approx\left[\mathcal{E}_{x}(0) e^{i \phi(0)}+g \ell \epsilon(0) \mathcal{E}_{y}(0)\right] e^{i(k \ell-\omega t)} .
$$

Employing the expression for the ellipticity, Eq. (5), in Eq. (7), we obtain

$$
E_{x}^{+}(\ell) \approx \mathcal{E}_{x}(0) e^{i(k \ell-\omega t)}\left[e^{i \phi(0)}+g \ell \sin \phi(0)\right] .
$$

In order to describe squeezing of vacuum fluctuations, one must use a quantum-mechanical description of the light field. We introduce the quantum-mechanical operator $\hat{E}_{x}$ for a monochromatic $x$-polarized light field, which can be written in terms of the photon annihilation and creation operators, $\hat{a}_{x}$ and $\hat{a}_{x}^{\dagger}$, respectively, as (see, e.g., Ref. [3])

$$
\hat{E}_{x}=\frac{\mathcal{E}_{0}}{2}\left[\hat{a}_{x} e^{i(k z-\omega t)}+\hat{a}_{x}^{\dagger} e^{-i(k z-\omega t)}\right]
$$

where $\mathcal{E}_{0}$ is the characteristic amplitude of unsqueezed vacuum fluctuations $[2-4,6]$. The creation and annihilation operators satisfy the commutation relations

$$
\begin{gathered}
{\left[\hat{a}_{\lambda}, \hat{a}_{\lambda^{\prime}}^{\dagger}\right]=\delta\left(\lambda, \lambda^{\prime}\right)} \\
{\left[\hat{a}_{\lambda}, \hat{a}_{\lambda^{\prime}}\right]=0,}
\end{gathered}
$$

where $\lambda$ refers to the mode (light frequency and polarization) and $\delta\left(\lambda, \lambda^{\prime}\right)$ is the Kronecker-delta symbol. When one is interested in the behavior of a multimode field, one can carry out a sum or integral over the appropriate modes (see, e.g., Refs. [6,26]). Such an approach is important in situations in which the squeezing is frequency dependent over the bandwidth of the light. Here, we assume that SR does not vary over the bandwidth of the light and that the measurement time is much longer than the inverse of the spectral width of the SR features, so for simplicity, we consider a single-mode field and make use of Eq. (9).

Since the degree of squeezing is phase dependent, in an experiment it is necessary to use a phase-sensitive detection technique (such as a balanced-homodyne scheme [3]). In such a scheme one measures the electromagnetic field at a particular phase $\chi$ with respect to a local oscillator. The phase-dependent electric-field operator (see, e.g., Ref. [3]) is given by

$$
\hat{E}_{x}(\chi, z)=\frac{\mathcal{E}_{0}}{2}\left[\hat{a}_{x}(z) e^{i \chi}+\hat{a}_{x}^{\dagger}(z) e^{-i \chi}\right],
$$

where we have assumed that the frequency of the local oscillator is the same as that of the monochromatic light wave, and $\chi$ is the time-independent phase difference between the local oscillator and the electromagnetic field at the output.

In analogy with the classical formulas for ellipticity, Eqs. (4) and (5), we introduce an ellipticity operator $\hat{\epsilon}$ for a nearly $y$-polarized light beam:

$$
\hat{\boldsymbol{\epsilon}}(z)=\mathcal{E}_{0} \frac{\hat{a}_{x}(z)-\hat{a}_{x}^{\dagger}(z)}{2 i \mathcal{E}_{y}(z)},
$$

where $\hat{a}_{x}(z)$ and $\hat{a}_{x}^{\dagger}(z)$ are the photon annihilation and creation operators at position $z$. This operator gives the ratio of 
the component of $E_{x}$ out-of-phase with $E_{y}$ to $E_{y}$. Indeed, supposing that the $x$-polarized field component is a coherent state $|\eta\rangle$ (which is an eigenstate of the annihilation operator with eigenvalue $\eta=|\eta| e^{i \phi}$ ) at position $z$, we can use the above operator to reproduce Eq. (5):

$$
\begin{aligned}
\boldsymbol{\epsilon} & =\langle\hat{\boldsymbol{\epsilon}}\rangle \\
& =\left\langle\eta\left|\frac{\mathcal{E}_{0}\left[\hat{a}_{x}(z)-\hat{a}_{x}^{\dagger}(z)\right]}{2 i \mathcal{E}_{y}(z)}\right| \eta\right\rangle \\
& =\frac{\mathcal{E}_{0}}{2 i \mathcal{E}_{y}(z)}\left(\eta-\eta^{*}\right)=\frac{|\eta| \mathcal{E}_{0}}{\mathcal{E}_{y}(z)} \sin \phi(z)=\frac{\mathcal{E}_{x}(z)}{\mathcal{E}_{y}(z)} \sin \phi(z) .
\end{aligned}
$$

In analogy to Eq. (7), we have for the $x$-polarized optical electric field after propagation through the vapor of length $\ell$

$$
\hat{E}_{x}^{+}(\ell)=\frac{\mathcal{E}_{0}}{2}\left\{\hat{a}_{x}(0)+\frac{i g \ell}{2}\left[\hat{a}_{x}^{\dagger}(0)-\hat{a}_{x}(0)\right]\right\} e^{i \chi} .
$$

It is useful to write the output field operator $\hat{E}_{x}(\ell)$ in terms of its own set of creation and annihilation operators as in Eq. (11), in which case the annihilation operator $\hat{a}_{x}(\ell)$ at the output of the medium is given by

$$
\hat{a}_{x}(\ell)=\hat{a}_{x}(0)+\frac{i g \ell}{2}\left[\hat{a}_{x}^{\dagger}(0)-\hat{a}_{x}(0)\right]
$$

Let us calculate what happens to the $x$-polarized vacuum field when light that is linearly polarized along $y$ propagates through the atomic vapor, assuming that there is no absorption $(\alpha=0)$. By using our expression for $\hat{a}_{x}(\ell)$ from Eq. (15) in Eq. (11), we find that the phase-dependent operator describing the $x$-polarized field after propagation through the atomic medium is

$$
\begin{aligned}
\hat{E}_{x}(\chi, \ell)= & \frac{\mathcal{E}_{0}}{2} \hat{a}_{x}(0)\left(e^{i \chi}-i g \ell \cos \chi\right) \\
& +\frac{\mathcal{E}_{0}}{2} \hat{a}_{x}^{\dagger}(0)\left(e^{-i \chi}+i g \ell \cos \chi\right) .
\end{aligned}
$$

If the input $x$-polarized field is the vacuum state $|0\rangle$, then the expectation value of the output field $\left\langle\hat{E}_{x}(\chi, \ell)\right\rangle=0$. However, $\left\langle\hat{E}_{x}(\chi, \ell)^{2}\right\rangle$ is nonzero, so the quantum fluctuations in $E_{x}(\chi, \ell)$, given by

$$
\begin{aligned}
\left\langle\Delta E_{x}(\chi, \ell)^{2}\right\rangle & =\left\langle\hat{E}_{x}(\chi, \ell)^{2}\right\rangle-\left\langle\hat{E}_{x}(x, \ell)\right\rangle^{2}=\left\langle\hat{E}_{x}(\chi, \ell)^{2}\right\rangle \\
& =\frac{\mathcal{E}_{0}^{2}}{4}\left(1-2 g \ell \sin \chi \cos \chi+g^{2} \ell^{2} \cos ^{2} \chi\right)
\end{aligned}
$$

are phase dependent, and for a particular choice of the phase $\chi$ can be made smaller than the fluctuations of the vacuum field $\left(\mathcal{E}_{0}^{2} / 4\right)$. Figure 1 shows the quantum fluctuations $\left\langle\Delta E_{x}^{2}\right\rangle /\left(\mathcal{E}_{0}^{2} / 4\right)[$ Eq. (17)] as a function of $\chi$ for the case of $g \ell=5$, illustrating that there is a restricted range of phases for which large squeezing is obtained.

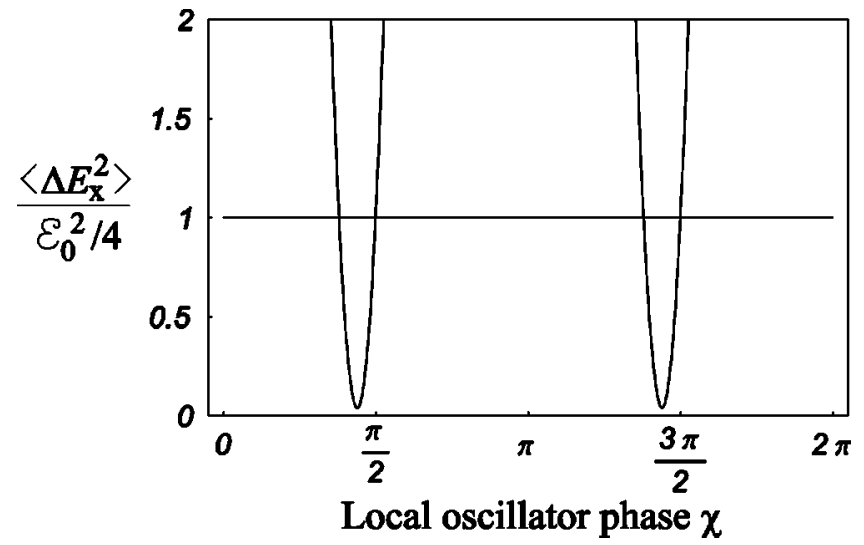

FIG. 1. Quantum fluctuations $\left\langle\Delta E_{x}^{2}\right\rangle /\left(\mathcal{E}_{0}^{2} / 4\right)$ as a function of the local oscillator phase $\chi$ for the case of zero absorption $(\alpha=0)$ and $g \ell=5$.

The optimum phase of the local oscillator for squeezing is found by setting

$$
\frac{\partial}{\partial \chi}\left\langle\left[\Delta E_{x}(\chi, \ell)\right]^{2}\right\rangle=0
$$

We have

$$
2 \cos 2 \chi+g \ell \sin 2 \chi=0,
$$

so that the optimum phase $\chi_{\mathrm{opt}}$ is

$$
\chi_{\mathrm{opt}}=\frac{1}{2} \arctan \left(-\frac{2}{g \ell}\right)+\left(n+\frac{1}{2}\right) \pi
$$

where

$$
n=1,2,3, \ldots
$$

The minimum fluctuations in the $x$-polarized output field are

$$
\left\langle\left[\Delta E_{x}\left(\chi_{\mathrm{opt}}, \ell\right)\right]^{2}\right\rangle=\frac{\mathcal{E}_{0}^{2}}{4}\left[1+\frac{g^{2} \ell^{2}}{2}-\frac{2+g^{2} \ell^{2} / 2}{\sqrt{1+4 /\left(g^{2} \ell^{2}\right)}}\right] .
$$

In the limit where $g \ell \gg 1$, we obtain

$$
\left\langle\left[\Delta E_{x}\left(\chi_{\mathrm{opt}}, \ell\right)\right]^{2}\right\rangle \approx \frac{\mathcal{E}_{0}^{2}}{g^{2} \ell^{2}} .
$$

In general, since the creation and annihilation operators do not commute [Eq. (10)], there is an uncertainty relationship between the fluctuations measured at a local oscillator phase of $\chi$ and a phase $\chi+\pi / 2$ (see, e.g., Ref. [3]):

$$
\left\langle\left[\Delta E_{x}(\chi, \ell)\right]^{2}\right\rangle\left\langle\left[\Delta E_{x}(\chi+\pi / 2, \ell)\right]^{2}\right\rangle \geqslant\left(\frac{\mathcal{E}_{0}^{2}}{4}\right)^{2} .
$$

If Eq. (24) is satisfied as an equality for some phase $\chi$, then the electromagnetic field is said to be in a minimumuncertainty state. In this sense, the squeezed vacuum state 
produced by SR is a minimum-uncertainty state, as can be seen by choosing $\chi=\chi_{\text {opt }}$ in Eq. (24).

We now turn to SR media that have nonzero absorption. One can model a medium with small total absorption in the following way. We assume that we have an ideal transparent SR medium. A small fraction of the light transmitted through the medium is reflected away by a beam splitter. At the same time, vacuum fluctuations enter through the dark port of the beamsplitter. Thus the squeezed vacuum is attenuated by an amount $e^{-\alpha \ell} \approx 1-\alpha \ell$ while $1-e^{\alpha \ell} \approx \alpha \ell$ of noise is added in quadrature. Equation (23) becomes

$$
\left\langle\left[\Delta E_{x}\left(\chi_{\mathrm{opt}}, \ell\right)\right]^{2}\right\rangle \approx \frac{\mathcal{E}_{0}^{2}}{4}\left(\frac{4}{g^{2} \ell^{2}}+\alpha \ell\right) .
$$

We would like to optimize squeezing with respect to $\ell$, so we set

$$
\frac{\partial}{\partial \ell}\left\langle\left[\Delta E_{x}\left(\chi_{\mathrm{opt}}, \ell\right)\right]^{2}\right\rangle=0,
$$

from which we obtain the optimum number of absorption lengths

$$
\alpha \ell_{\mathrm{opt}}=2\left(\frac{\alpha}{g}\right)^{2 / 3}
$$

Substituting the value of $\ell_{\text {opt }}$ from Eq. (27) into Eq. (25) and taking the limit where $g / \alpha \gg 1$, we obtain

$$
\left\langle\left[\Delta E_{x}\left(\chi_{\text {opt }}, \ell_{\text {opt }}\right)\right]^{2}\right\rangle \approx \frac{3 \mathcal{E}_{0}^{2}}{4}\left(\frac{\alpha}{g}\right)^{2 / 3} .
$$

The squeezing parameter $s$ is the ratio of the amplitudes of the quantum fluctuations after and before light propagates through the SR medium:

$$
s=\sqrt{\frac{\mathcal{E}_{0}^{2} / 4}{\left\langle\left[\Delta E_{x}\left(\chi_{\mathrm{opt}}, \ell_{\mathrm{opt}}\right)\right]^{2}\right\rangle}} .
$$

The reason that $s$ is the relevant parameter describing squeezing is that in order to take advantage of the reduced noise in the vacuum field, one must interfere the squeezed vacuum with another field of nonzero amplitude. Then the dominant noise contribution due to the vacuum appears as a cross term between the real and vacuum field amplitudes. In such a scenario, $s$ represents the improvement in the signalto-noise ratio over the shot-noise limit.

From Eq. (28), we find

$$
s=\frac{1}{\sqrt{3}}\left(\frac{g}{\alpha}\right)^{1 / 3} .
$$

Equation (30) represents a general result for the optimal (with respect to local oscillator phase $\chi$ and path length $\ell$ ) vacuum squeezing obtainable in an SR medium with small total absorption.

In this article, numerical values for $s$ are given in $\mathrm{dB}$, obtained by taking $10 \log _{10} s$.
Often, it is useful to characterize the light field in terms of the Stokes parameters, given in terms of the positive- and negative-frequency components of the field by (see, e.g., Ref. [29])

$$
\begin{gathered}
S_{0}=E_{x}^{+} E_{x}^{-}+E_{y}^{+} E_{y}^{-} \approx\left|\mathcal{E}_{y}\right|^{2}, \\
S_{1}=E_{x}^{+} E_{x}^{-}-E_{y}^{+} E_{y}^{-} \approx-\left|\mathcal{E}_{y}\right|^{2}, \\
S_{2}=E_{x}^{+} E_{y}^{-}+E_{x}^{-} E_{y}^{+} \approx \mathcal{E}_{y}\left(E_{x}^{+}+E_{x}^{-}\right), \\
S_{3}=i\left(E_{x}^{+} E_{y}^{-}-E_{x}^{-} E_{y}^{+}\right) \approx i \mathcal{E}_{y}\left(E_{x}^{+}-E_{x}^{-}\right),
\end{gathered}
$$

so, in the case considered here, we obtain squeezing in particular combinations of the $S_{2}$ and $S_{3}$ Stokes parameters.

\section{DESCRIPTION OF THE DENSITY-MATRIX CALCULATION}

In order to determine the vacuum squeezing produced by a given atomic system, we perform a calculation of the parameters $g$ and $\alpha$ based on a standard density matrix approach. The time evolution of the atomic density-matrix $\rho$ under the action of the light-atom interaction Hamiltonian $H_{L}=-\mathbf{E} \cdot \mathbf{d}$, where $\mathbf{E}$ is the electric-field vector, and $\mathbf{d}$ is the dipole operator, is given by the Liouville equation (see, e.g., Ref. [27])

$$
\frac{d \rho}{d t}=\frac{1}{i \hbar}[H, \rho]-\frac{1}{2}\{\Gamma, \rho\}+\Lambda,
$$

where the square brackets denote the commutator and the curly brackets the anticommutator, and the total Hamiltonian $H$ is the sum of $H_{L}$ and the unperturbed Hamiltonian $H_{0} . \Gamma$ is the relaxation matrix (diagonal in the collision-free approximation)

$$
\langle\xi J m|\Gamma| \xi J m\rangle=\gamma+\gamma_{0} \delta\left(\xi, \xi_{e}\right),
$$

where $\gamma$ and $\gamma_{0}$ are the ground-state depolarization rate and the spontaneous decay rate from the upper state, respectively, and $\xi$ represents the quantum number distinguishing the ground state $\xi_{g}$ from the excited state $\xi_{e} \cdot \Lambda=\Lambda^{0}+\Lambda^{\text {repop }}$ is the pumping term, where the diagonal matrix

$$
\left\langle\xi_{g} J_{g} m\left|\Lambda^{0}\right| \xi_{g} J_{g} m\right\rangle=\frac{\gamma \rho_{0}}{\left(2 J_{g}+1\right)}
$$

describes incoherent ground-state pumping ( $\rho_{0}$ is the atomic density), and

$$
\begin{aligned}
& \left\langle\xi_{g} J_{g} m\left|\Lambda^{\text {repop }}\right| \xi_{g} J_{g} m^{\prime}\right\rangle \\
& \quad=\gamma_{0} \sum_{m_{e}, m_{e}^{\prime}, q}\left\langle J_{g}, m, 1, q \mid J_{e}, m_{e}\right\rangle\left\langle J_{g}, m^{\prime}, 1, q \mid J_{e}, m_{e}^{\prime}\right\rangle \\
& \quad \times \rho_{\xi_{e}} J_{e} m_{e} \xi_{e} J_{e} m_{e}^{\prime},
\end{aligned}
$$

describes repopulation due to spontaneous relaxation from the upper level (see, e.g., Ref. [28]). Here $\langle\ldots \mid \ldots\rangle$ are the Clebsch-Gordan coefficients. 


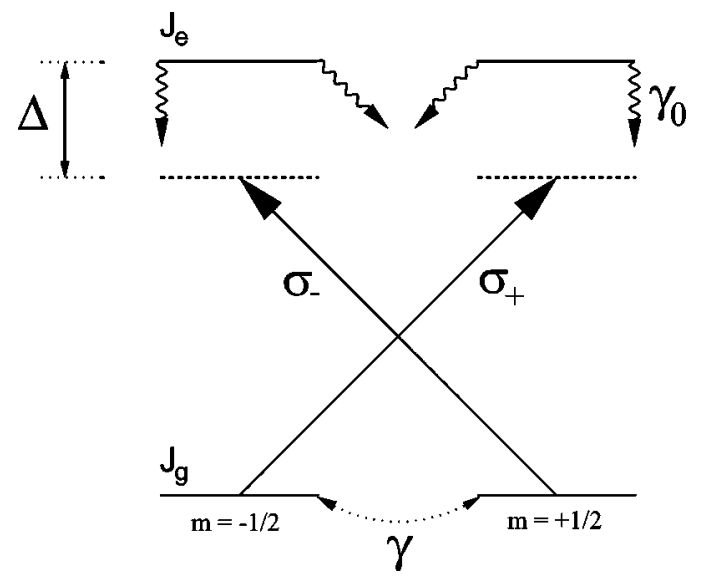

FIG. 2. Schematic energy level diagram for the X-system $\left(J_{g}\right.$ $=1 / 2 \rightarrow J_{e}=1 / 2$ transition). $\gamma$ denotes the relaxation rate of groundstate atomic polarization; $\gamma_{0}$ denotes the homogeneous width of the upper state; $\sigma_{+}$and $\sigma_{-}$represent the left- and right-circularly polarized components of the light field, whose frequency is detuned by $\Delta$ from resonance.

The electric-field vector is written (see, e.g., Ref. [29])

$$
\begin{aligned}
\mathbf{E}= & \frac{1}{2}\left[E_{0} e^{i \phi}(\cos \varphi \cos \epsilon-i \sin \varphi \sin \epsilon) e^{i(\omega t-k z)}+\text { c.c. }\right] \hat{\mathbf{x}} \\
& +\frac{1}{2}\left[E_{0} e^{i \phi}(\sin \varphi \cos \epsilon+i \cos \varphi \sin \epsilon) e^{i(\omega t-k z)}+\text { c.c. }\right] \hat{\mathbf{y}}
\end{aligned}
$$

where $E_{0}$ is the electric-field amplitude, $\varphi$ is the polarization angle, and $\phi$ is the overall phase. By substituting Eq. (36) into the wave equation

$$
\left(\frac{\omega^{2}}{c^{2}}+\frac{d^{2}}{d z^{2}}\right) \mathbf{E}=-\frac{4 \pi}{c^{2}} \frac{d^{2}}{d t^{2}} \mathbf{P},
$$

where $\mathbf{P}=\operatorname{Tr} \rho \mathbf{d}$ is the polarization of the medium, the absorption, rotation, phase shift, and change of ellipticity per unit distance for an optically thin medium can be found in terms of the density-matrix elements (these expressions are given in Ref. [11]).

\section{ANALYSIS FOR $J_{g}=1 / 2 \rightarrow J_{e}=1 / 2,3 / 2$ SYSTEMS}

Using the rotating-wave approximation, we solve Eq. (32) for a closed $J_{g}=1 / 2 \rightarrow J_{e}=1 / 2$ transition (an $X$ system, Fig. 2 ); analytic solutions for the optical response are readily obtained in this case. We assume homogeneous broadening, since, as will be shown later, the homogeneous width due to power broadening exceeds the Doppler width under the conditions where large vacuum squeezing is obtained. This simple system can be realized experimentally in a number of different ways. For example, at light powers high enough so that hyperfine effects can be neglected, the $D 1$ line in alkali atoms can be treated as a simple $1 / 2 \rightarrow 1 / 2$ system. Also, SR in this system was studied theoretically and experimentally in Ref. [12], where $\mathrm{K}$ atoms were employed and He buffer gas was used to collisionally broaden the $4^{2} S_{1 / 2} \rightarrow 4^{2} P_{1 / 2}$ transition to remove hyperfine effects.

The solutions can be simplified by assuming that $\gamma$ $\ll \gamma_{0}$. For the absorption coefficient $\alpha$ as a function of light frequency detuning $\Delta$ and in terms of the optical-pumping saturation parameter

$$
\kappa=\frac{d^{2} E_{0}^{2}}{\gamma \gamma_{0}}
$$

where $d$ is the reduced electric-dipole matrix element, we find

$$
\alpha(\kappa, \Delta) \approx \frac{\alpha_{0}}{1+4\left(\Delta / \gamma_{0}\right)^{2}+\left(\gamma / \gamma_{0}\right)(\kappa / 3)},
$$

where $\alpha_{0}=\alpha(0,0)$ is the unsaturated absorption coefficient on resonance, given by

$$
\alpha_{0}=\frac{n}{2 \pi} \lambda^{2} \frac{2 J_{e}+1}{2 J_{g}+1}
$$

where $n$ is the atomic density and $\lambda$ is the wavelength of the light. As a function of $\Delta, \alpha(\kappa, \Delta)$ is a power-broadened Lorentzian profile. For the self-rotation parameter $g$, we find

$$
g(\kappa, \Delta) \approx \frac{2}{9} \frac{\alpha(\kappa, \Delta) \kappa \Delta / \gamma_{0}}{1+4\left(\Delta / \gamma_{0}\right)^{2}+\kappa / 9} .
$$

From Eq. (41), we see that at low light powers $(\kappa \ll 1)$, SR increases linearly with light intensity. At high light intensities ( $\kappa \gg 1), g$ falls off as $\kappa^{-1}$. We also note that while $\alpha$ is an even function of detuning, $g$ is antisymmetric with respect to $\Delta$.

From these expressions we find the squeezing $s$ [Eq. (30)], optimized in terms of the sample length $\ell$ and the local oscillator phase $\chi$,

$$
s(\kappa, \Delta) \approx \frac{1}{3}\left[\frac{2}{\sqrt{3}} \frac{\kappa \Delta / \gamma_{0}}{1+4\left(\Delta / \gamma_{0}\right)^{2}+\kappa / 9}\right]^{1 / 3} .
$$

The detuning that maximizes $s$ for a given $\kappa$ is

$$
\Delta_{\mathrm{opt}}=\frac{\gamma_{0}}{2} \sqrt{1+\kappa / 9}
$$

The optimum detuning increases with light intensity due to power broadening. Substituting $\Delta_{\text {opt }}$ into Eq. (42) and taking the limit of large light power gives the best possible squeezing as a function of light power:

$$
s\left(\kappa, \Delta_{\mathrm{opt}}\right) \approx\left(\frac{\kappa}{972}\right)^{1 / 6}=\left(\frac{d^{2} E_{0}^{2}}{972 \gamma_{0} \gamma}\right)^{1 / 6} .
$$

The number of unsaturated absorption lengths on resonance required to produce this squeezing can be obtained using Eq. (27)

$$
\alpha_{0} \ell_{\mathrm{opt}} \approx 2\left[\frac{\alpha\left(\kappa, \Delta_{\mathrm{opt}}\right)}{g\left(\kappa, \Delta_{\mathrm{opt}}\right)}\right]^{2 / 3} \frac{\alpha_{0}}{\alpha\left(\kappa, \Delta_{\mathrm{opt}}\right)} \approx\left(\frac{4 \sqrt{2}}{9} \kappa\right)^{2 / 3} .
$$


Performing a similar analysis for a closed $J_{g}=1 / 2 \rightarrow J_{e}$ $=3 / 2$ transition, corresponding to the D2 line in alkali atoms, one obtains

$$
\begin{gathered}
s\left(\kappa, \Delta_{\mathrm{opt}}\right) \approx\left(\frac{\kappa}{7776}\right)^{1 / 6} \\
\alpha_{0} \ell_{\mathrm{opt}} \approx\left(\frac{4 \sqrt{2}}{9} \kappa\right)^{2 / 3},
\end{gathered}
$$

which is, up to a numerical factor for $s$, the same as for the X-system.

An important assumption in our derivation of Eq. (30) was that the ellipticity of the light does not change significantly as the light propagates through the atomic medium. Now we can justify this assumption for the cases considered in the present section. There are two mechanisms which can cause "self-elliptization" (SE) of the light field: linear birefringence and circular dichroism. In general, because dichroic effects are related to the absorptive properties of the medium and we are always in a regime where $\alpha \ell$ is small, $\mathrm{SE}$ caused by circular dichroism is negligible. In Ref. [11], there are two physical mechanisms identified as possible causes of SR for systems with $J_{g}=1 / 2$ : optical pumping and ac Stark shifts, both of which lead only to circular dichroism. Thus SE can be neglected in the present cases. For the $\mathrm{X}$-system, the change in ellipticity $\delta \epsilon$ is described by

$$
\frac{\delta \epsilon}{\epsilon(0) \ell} \approx \frac{2}{9} \frac{\alpha(\kappa, \Delta) \kappa}{1+4\left(\Delta / \gamma_{0}\right)^{2}+\kappa / 9} .
$$

We see that SE is suppressed by a factor of $\gamma_{0} / \Delta$ compared to SR [Eq. (41)]. Under optimum conditions for squeezing (see Sec. V) and $\kappa \gg 1, \gamma_{0} / \Delta \sim 6 / \sqrt{\kappa}$-confirming that SE is negligible in our case. These arguments also show why in the present cases SE is not an effective mechanism for producing squeezed vacuum in one of the circular components of the light field.

\section{ESTIMATE OF ACHIEVABLE VACUUM SQUEEZING}

First we consider the vacuum squeezing attainable by using a buffer-gas-free cell without antirelaxation wall coating. Generally, for a given laser power, a smaller beam diameter results in higher $\kappa$ and better squeezing [30]. For a reasonably attainable $1-\mathrm{W}, 100-\mu \mathrm{m}$-diameter laser beam, the light electric-field amplitude is $\sim 3 \mathrm{kV} / \mathrm{cm}$ and the effective ground-state relaxation rate resulting from the transit of atoms through the laser beam is $\sim 3 \mathrm{MHz}$. For the $\mathrm{Rb} D 1$ line (which at these light intensities is effectively an X-system), $\kappa \approx 10^{8}$ and $s \approx 8.4 \mathrm{~dB}$ [Eq. (44), Fig. 3]. From Eq. (45), we see that to obtain this level of squeezing, one must use a relatively dense atomic vapor $\left(\alpha_{0} \ell \approx 2 \times 10^{5}\right.$, for a cell of length $10 \mathrm{~cm}$, this corresponds to a density of $\sim 10^{13}$ atoms $/ \mathrm{cm}^{3}$ ).

Various techniques can be considered to reduce the ground-state relaxation rate in order to produce larger $\kappa$, and therefore, in principle, improve vacuum squeezing. Cold atom traps (in particular far-off-resonant, blue-detuned opti-

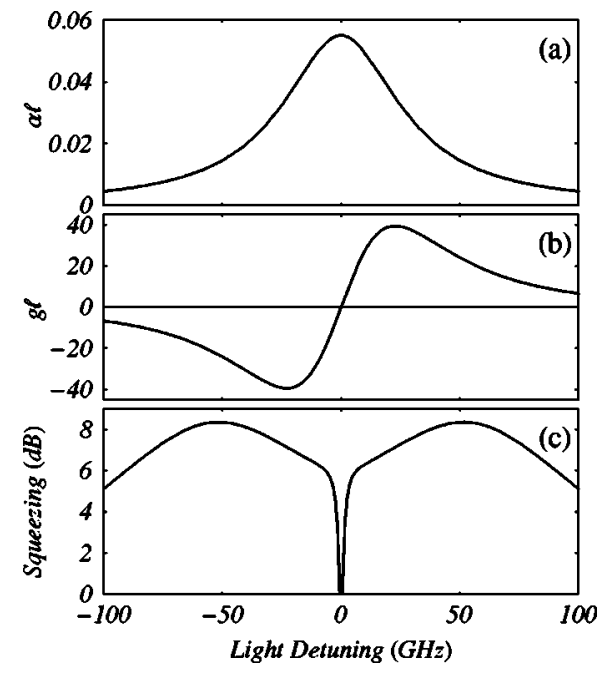

FIG. 3. Plots of (a) absorption coefficient $\alpha$ times path length $\ell$, (b) self-rotation parameter $g$ [Eq. (1)] times path length $\ell$, and (c) vacuum squeezing in $\mathrm{dB}$ for an $\mathrm{X}$-system $(\mathrm{Rb} D 1$ line at high light power in a buffer-gas-free, uncoated vapor cell). Light power is 1 $\mathrm{W}$, beam diameter is $100 \mu \mathrm{m}$, atomic density is $10^{13}$ atoms $/ \mathrm{cm}^{3}$, and cell length $\ell=10 \mathrm{~cm}$.

cal dipole traps) can produce alkali vapors with ground-state relaxation rates $\sim 0.1 \mathrm{~Hz}[31]$, but the densities achieved to date are too low to obtain the required optical thickness for optimum squeezing. In order to achieve $s \simeq 8 \mathrm{~dB}$ with cold alkali-metal atoms, for a $100-\mu \mathrm{m}$-long trap (with crosssectional area of $30 \mu \mathrm{m} \times 30 \mu \mathrm{m}$ ), one would need $\sim 10^{10}$ atoms in the trap, which is beyond present capabilities.

Alternatively, buffer gas can be used to reduce the ground-state relaxation rate by lengthening the transit time of atoms through the light beam. The transit time can be increased until the point where the diffusion rate equals the relaxation rate due to depolarizing collisions with the buffer gas. Under conditions where the transit time is determined by diffusion, the relaxation rate of ground-state polarization is

$$
\gamma^{\prime} \approx \frac{\mathcal{D}}{x^{2}}+a_{1} n_{b}
$$

where $x$ is the light beam diameter, $n_{b}$ is the buffer gas density, $a_{1}$ is a constant describing the rate of depolarizing collisions, and $\mathcal{D} \approx v /\left(3 n_{b} \sigma\right)$ is the diffusion coefficient ( $v$ is the average atomic velocity and $\sigma$ is the cross section for elastic collisions). In addition, one must also take into account the increase in the homogeneous width of the atomic transition due to pressure broadening. The homogeneous width is given by

$$
\gamma_{0}^{\prime}=\gamma_{0}+a_{2} n_{b}
$$

where $a_{2}$ is a constant describing the pressure broadening rate. Thus, for a given light power and beam diameter, the ratio of $\kappa^{\prime}=d^{2} E_{0}^{2} /\left(\gamma_{0}^{\prime} \gamma^{\prime}\right)$ to $\kappa$ for a buffer-gas-free cell [Eq. (38)] is 


$$
\frac{\kappa^{\prime}}{\kappa}=\frac{\gamma_{0} \gamma}{\gamma_{0}^{\prime} \gamma^{\prime}} \approx \frac{\gamma_{0} \gamma}{\left[v /\left(3 n_{b} \sigma x^{2}\right)+a_{1} n_{b}\right]\left(\gamma_{0}+a_{2} n_{b}\right)}
$$

where we note again that $\kappa^{\prime}$ is calculated in the diffusionlimited regime. We see from Eq. (50) that for $n_{b}$ too large, $\kappa^{\prime} / \kappa$ is less than one, so there is in fact an optimum buffergas pressure at which squeezing is maximized. For typical values of cross sections for the relaxation of atomic polarization in alkali-noble-gas collisions [32] and pressure broadening [33], we estimate the maximum achievable $\kappa^{\prime} / \kappa$ to be about 2-3. Thus buffer-gas-filled cells do not appear to allow substantial improvement in squeezing at high light powers.

Antirelaxation-coated vapor cells can also be used to drastically decrease the relaxation rate of ground-state atomic polarization in the alkali metals [34-39]. In order to obtain optimal squeezing in, e.g., a 10-cm-diameter paraffin-coated cell, estimates show that the required atomic density is $n$ $\gtrsim 10^{13}$ atoms $/ \mathrm{cm}^{3}$ [Eq. (45)]. In this high-density regime, $\gamma$ is primarily due to spin-exchange collisions between the alkali-metal atoms instead of collisions with the wall, which greatly diminishes the advantages of using paraffin coating. The relatively fast relaxation due to spin-exchange collisions significantly reduces the probability that an atom retains its polarization upon returning to the beam after traveling about the cell - thereby reducing SR related to coherence effects [11]. These factors indicate that one cannot generate significantly better squeezing via SR by using paraffin-coated cells.

Thus, at present, no system considered above offers significant improvement in vacuum squeezing via SR compared to buffer-gas-free, uncoated alkali-metal vapor cells. However, we note that improvements in the experimental techniques discussed may change this conclusion in the future.

\section{CALCULATION FOR THE ${ }^{87}$ Rb $D$ LINES}

Recent experiments $[11,13]$ have studied SR in $\mathrm{Rb}$ vapors at lower light intensities than considered in Sec. V. We have performed density-matrix calculations (Sec. III and Ref. [11]) for conditions achievable in these experiments. Since our formula for the squeezing parameter $s[\mathrm{Eq} .(30)]$ is based on the assumptions that $g \ell \gg 1$ and $\alpha \ell \ll 1$, we are restricted to light intensities and frequencies where these conditions are satisfied. In addition, our density-matrix calculation treats ground-state hyperfine levels individually. Thus the calculations are not valid for light intensities so high that the ground-state hyperfine structure is not resolved. In order to use the highest possible laser intensity in the calculation, we study the $D 1$ and $D 2$ lines of ${ }^{87} \mathrm{Rb}$, which has larger groundstate hyperfine separation than ${ }^{85} \mathrm{Rb}$. The results of these calculations are shown in Figs. 4, 5, and 6.

In Figs. 4 and 5, we choose laser power equal to $10 \mathrm{~mW}$ (readily obtainable with the tunable diode laser systems employed in Refs. $[11,13]$ ) and beam diameter $0.03 \mathrm{~cm}$, which results in the highest light intensity possible while still resolving the ground-state hyperfine lines $\left(\sim 10^{4} \mathrm{~mW} / \mathrm{cm}^{2}\right.$ [40]). Assuming a 10-cm-long vapor cell, we find the atomic density for which the squeezing is globally maximized (with respect to light detuning) by finding the light frequency where $g / \alpha$ is maximized, then fixing the density according to

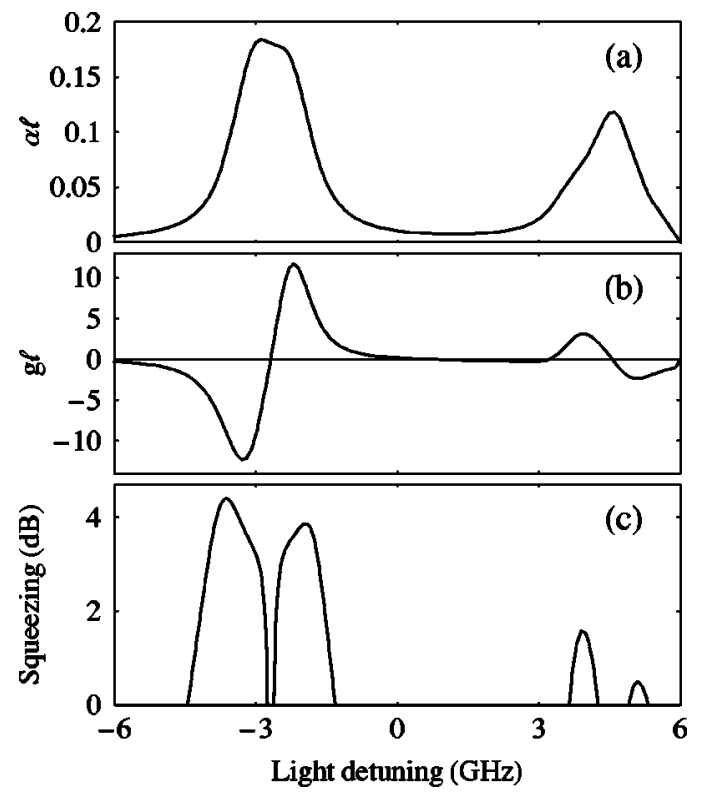

FIG. 4. Plots of (a) absorption coefficient $\alpha$ times path length $\ell$, (b) self-rotation parameter $g$ [Eq. (1)] times path length $\ell$, and (c) vacuum squeezing in $\mathrm{dB}$ for the ${ }^{87} \mathrm{Rb} D 1$ line. Atomic density is chosen to be $n=10^{12}$ atoms $/ \mathrm{cm}^{3}$ to obtain the maximum possible squeezing for the given light power $(10 \mathrm{~mW})$ and light beam diameter $(0.03 \mathrm{~cm})$, as described in text. The Doppler width is $2 \pi$ $\times 306 \mathrm{MHz}$.

Eq. (27). As can be seen, even with these modest parameters, squeezing of up to $6 \mathrm{~dB}$ can be obtained.

The density-matrix calculations also show that selfellipticity effects are small for the $D 1$ and $D 2$ lines of ${ }^{87} \mathrm{Rb}$

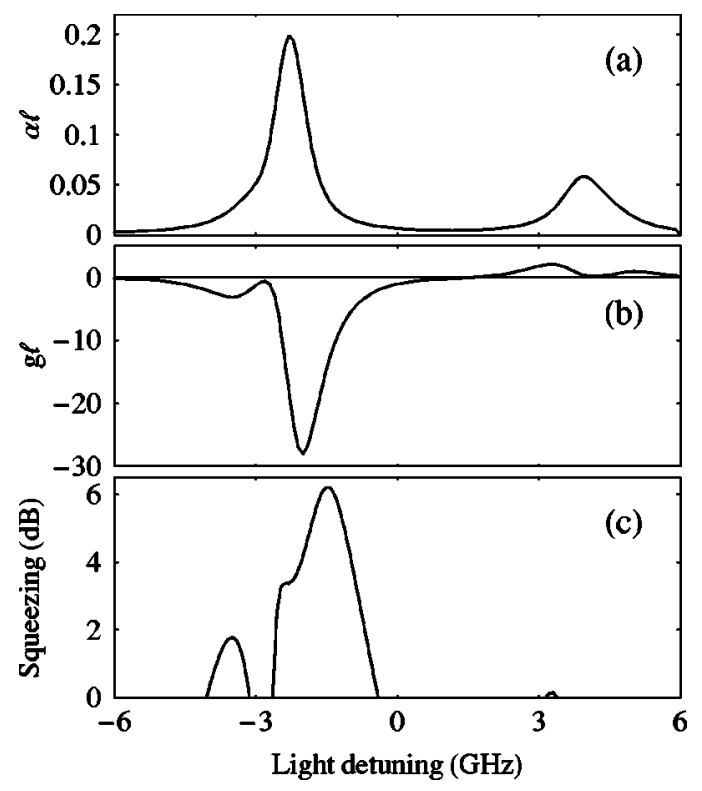

FIG. 5. Plots of (a) absorption coefficient times path length $(\alpha \ell)$, (b) self-rotation parameter times path length $(g \ell)$, and (c) vacuum squeezing in $\mathrm{dB}$ for the ${ }^{87} \mathrm{Rb} D 2$ line. Atomic density is chosen to be $n=2 \times 10^{11}$ atoms $/ \mathrm{cm}^{3}$ to maximize squeezing for the given light power $(10 \mathrm{~mW})$ and light beam diameter $(0.03 \mathrm{~cm})$, as described in text. The Doppler width is $2 \pi \times 306 \mathrm{MHz}$. 


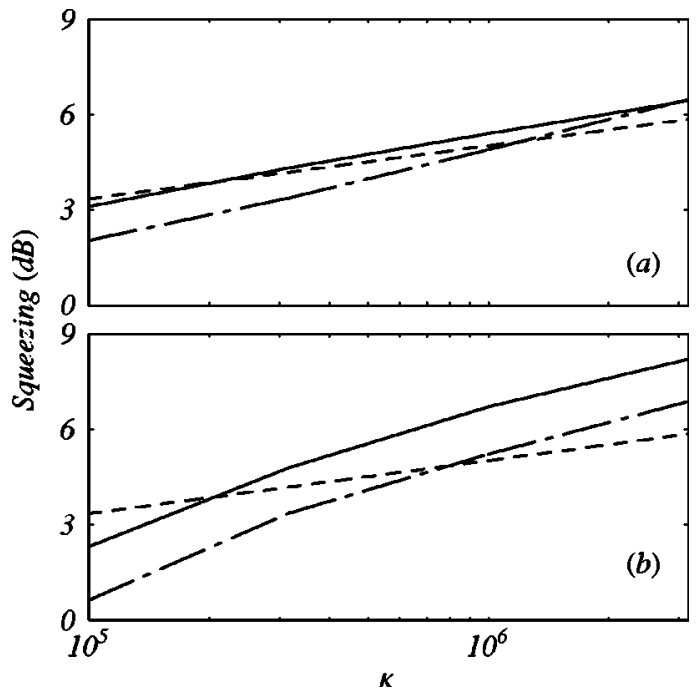

FIG. 6. Comparison of squeezing as a function of the saturation parameter $\kappa$ for the X-system (dashed line) to squeezing for the (a) ${ }^{87} \mathrm{Rb} \mathrm{D} 1$ line and (b) ${ }^{87} \mathrm{Rb} D 2$ line. The dot-dashed lines represent squeezing for $F=1 \rightarrow F^{\prime}$ transitions and the solid lines represent squeezing for $F=2 \rightarrow F^{\prime}$ transitions. The range of $\kappa$ is chosen so that the ground-state hyperfine levels can be treated independently, as discussed in the text.

under these conditions. This verifies that the assumptions in the derivation of Eq. (30) are satisfied.

At sufficiently high light intensities, the hyperfine effects can be neglected, and the $D 1$ line becomes an $\mathrm{X}$ system, while the $D 2$ line becomes a $J_{g}=1 / 2 \rightarrow J_{e}=3 / 2$ system, so the analysis of Sec. IV applies. The intermediate regime, where the hyperfine levels are not fully resolved but hyperfine effects cannot be neglected, is beyond the scope of the present work. Figure 6 shows the maximum squeezing as a function of $\kappa$ for the $\mathrm{X}$ system and the ${ }^{87} \mathrm{Rb} D 1$ and $D 2$ lines for a range of $\kappa$ where the $\mathrm{Rb}$ calculations are valid. It is interesting to note that, for this range of $\kappa$, the squeezing in $\mathrm{Rb}$ can be somewhat larger than that obtained for the $\mathrm{X}$ system.

The significant vacuum squeezing that can be obtained in the $\mathrm{Rb} D$ lines at these relatively low light powers indicates the presence of an enhancement mechanism for squeezing. One possible cause of this enhancement is the effect of ground-state atomic coherence, which is not present in the $J_{g}=1 / 2 \rightarrow J_{e}=1 / 2$ and $J_{g}=1 / 2 \rightarrow J_{e}=3 / 2$ systems considered in Sec. IV. Recent research on electromagnetically induced transparency and coherent population trapping has shown that it is possible to have large nonlinear couplings with very small absorption (see, e.g., Refs. [19,41,42]) in related systems. Thus, according to Eq. (30), one could expect that systems where ground-state atomic coherence played a significant role would be favorable for squeezing. There are also SR mechanisms involving multiple hyperfine transitions [11] that play a role in the $\mathrm{Rb} D$ lines at low light power which are not present for the systems considered in Sec. IV. Further analysis is required to determine the exact physical mechanism for enhancement of squeezing in this particular case. Recently [43], vacuum squeezing via SR was analyzed for the double- $\Lambda$ system, a model system in which atomic co- herence and interference effects, as well as multitransition effects, play important roles.

Stimulated Raman scattering (SRS) may limit the light intensity which can be used, since for sufficiently high light intensities, SRS can convert a significant portion of the incident light power into new frequencies. In addition to the depletion of the input light beam, nonlinear instabilities associated with SRS may introduce extra noise. Estimates indicate that the parameters employed in the calculations yielding Figs. 4 and 5 are in the regime where SRS becomes significant, so additional analysis is required to determine the manner in which SRS affects the obtainable squeezing.

In a complete analysis of the noise properties of the light field, in addition to the absorptive properties of the atomic medium, one must account for the effect of the quantum noise of atomic states (see, e.g., Refs. [44,45]). Estimates indicate that in the high light power, high atomic density regime where the best vacuum squeezing is obtained (see, also, Sec. V), noise related to these effects is small enough that it should not degrade the predicted squeezing.

\section{CONCLUSION}

We have shown that when linearly polarized light propagates through a medium in which there is nonlinear selfrotation of elliptically polarized light, the vacuum electromagnetic field in the orthogonal polarization is squeezed. We have derived a simple expression [Eq. (30)] for the ratio of the amplitude of vacuum fluctuations at the output of the medium to those at the input under optimum conditions. Density-matrix calculations performed for relatively simple atomic systems indicate that under realistic conditions it should be possible to achieve $s \approx 8 \mathrm{~dB}$. We have compared various experimental systems, such as trapped and cooled atoms and buffer-gas-filled cells, finding that with presently achievable experimental parameters, none of the considered techniques offer substantial improvement over buffer-gasfree vapor cells. We have also performed calculations showing that vacuum squeezing of up to $6 \mathrm{~dB}$ should be achievable in vapors of ${ }^{87} \mathrm{Rb}$ with the experimental setups used in Refs. [11,13].

Squeezed vacuum allows one to surpass the short-noise limit in polarimetry [8]. The proposed technique is a simple way to generate squeezed vacuum at light wavelengths useful for a variety of applications, such as magnetometry [37$39,46,47]$ and discrete symmetry tests in atomic systems [48-50].

\section{ACKNOWLEDGMENTS}

The authors gratefully acknowledge the support from the Office of Naval Research, the National Science Foundation, and the Welch Foundation. A.M. acknowledges helpful discussions with M.O. Scully, M. Fleischhauer and A.S. Zibrov; D.B., S.M.R. and D.F.K. would like to thank D. Gauthier, V. Yashchuk, and M. Zolotorev. 
[1] C. M. Caves, Phys. Rev. D 23, 1693 (1981).

[2] D. F. Walls, Nature (London) 306, 141 (1983).

[3] R. Loudon and P. L. Knight, J. Mod. Opt. 34, 709 (1987).

[4] G. Leuchs, Contemp. Phys. 29, 299 (1988).

[5] D. F. Walls and G. J. Milburn, Quantum Optics (SpringerVerlag, Berlin, 1995).

[6] M. O. Scully and M. S. Zubairy, Quantum Optics (Cambridge University Press, Cambridge, 1997).

[7] M. Xiao, L.-A. Wu, and H. J. Kimble, Phys. Rev. Lett. 59, 278 (1987).

[8] P. Grangier, R. E. Slusher, B. Yurke, and A. LaPorta, Phys. Rev. Lett. 59, 2153 (1987).

[9] J. Opt. Soc. Am. B 4, 1450 (1987); Appl. Phys. B: Photophys. Laser Chem. 55, 189 (1992).

[10] P. D. Maker, R. W. Terhune, and C. M. Savage, Phys. Rev. Lett. 12, 507 (1964).

[11] S. M. Rochester, D. S. Hsiung, D. Budker, R. Y. Chiao, D. F. Kimball, and V. V. Yashchuk, Phys. Rev. A 63, 043814 (2001).

[12] W. V. Davis, A. L. Gaeta, and R. W. Boyd, Opt. Lett. 17, 1304 (1992).

[13] I. Novikova, A. B. Matsko, V. A. Sautenkov, V. L. Velichansky, G. R. Welch, and M. O. Scully, Opt. Lett. 25, 1651 (2000).

[14] I. Novikova, A. B. Matsko, V. L. Velichansky, M. O. Scully, and G. R. Welch, Phys. Rev. A 63, 063802 (2001).

[15] R. Tanas and S. Kielich, Opt. Commun. 45, 351 (1983); R. Tanas and S. Kielich, Opt. Acta 31, 81 (1984); S. Kielich, R. Tanas, and R. Zawodny, J. Opt. Soc. Am. B 4, 1627 (1987); S. Kielich, R. Tanas, and R. Zawodny, Phys. Rev. A 36, 5670 (1987); S. Kielich, R. Tanas, and R. Zawodny, Appl. Phys. B: Photophys. Laser Chem. 45, 249 (1988); R. Tanas and S. Kielich, J. Mod. Opt. 37, 1935 (1990).

[16] M. Margalit, C. X. Yu, E. P. Ippen, and H. A. Haus, Opt. Express 2, 72 (1998); L. Boivin and H. A. Haus, Opt. Lett. 21, 146 (1996).

[17] K. Hakuta, L. Marmet, and B. P. Stoicheff, Phys. Rev. Lett. 66, 596 (1991); S. E. Harris, J. E. Field, and A. Imamoglu, ibid. 64, 1107 (1990); A. Kasapi, M. Jain, G. Y. Yin, and S. E. Harris, ibid. 74, 2447 (1995); for a review, see S. E. Harris, Phys. Today 50(7), 36 (1997).

[18] P. R. Hemmer, D. P. Katz, J. Donoghue, M. Cronin-Golomb, M. S. Shahriar, and P. Kumar, Opt. Lett. 20, 982 (1995).

[19] M. D. Lukin, P. Hemmer, M. Loeffler, and M. O. Scully, Phys. Rev. Lett. 81, 2675 (1998).

[20] A. S. Zibrov, M. D. Lukin, and M. O. Scully, Phys. Rev. Lett. 83, 4049 (1999).

[21] M. D. Lukin, A. B. Matsko, M. Fleischhauer, and M. O. Scully, Phys. Rev. Lett. 82, 1847 (1999).

[22] M. Fleischhauer, M. D. Lukin, A. B. Matsko, and M. O. Scully, Phys. Rev. Lett. 84, 3558 (2000).

[23] H. Schmidt and A. Imamoğlu, Opt. Lett. 21, 1936 (1996); A. Imamoğlu, H. Schmidt, G. Woods, and M. Deutsch, Phys. Rev. Lett. 79, 1467 (1997); H. Schmidt and A. Imamoğlu, Opt. Lett. 23, 1007 (1998).

[24] K. M. Gheri, W. Alge, and P. Grangier, Phys. Rev. A 60, R2673 (1999).

[25] S. Harris and Y. Yamamoto, Phys. Rev. Lett. 81, 3611 (1998).

[26] C. M. Caves and B. L. Schumaker, Phys. Rev. A 31, 3068 (1985); ibid. 31, 3093 (1985).
[27] S. Stenholm, Foundations of Laser Spectroscopy (Wiley, New York, 1984).

[28] S. G. Rautian and A. M. Shalagin, Kinetic problems of nonlinear spectroscopy (North-Holland, New York, 1994).

[29] S. Huard, Polarization of Light (Wiley, New York, 1997).

[30] From Eqs. (44) and (46), one can see that for $J_{g}=1 / 2 \rightarrow J_{e}$ $=1 / 2$ and $J_{g}=1 / 2 \rightarrow J_{e}=3 / 2$ transitions, the higher the value of $\kappa$ [Eq. (38)], the better the squeezing. It turns out that for all the cases considered in this section, decreasing the light beam diameter either leaves $\kappa$ unchanged or makes it larger. This can be seen as follows. For a given light power, $E_{0}^{2}$ scales as $x^{-2}$, where $x$ is the light beam diameter. The homogeneous width $\gamma_{0}$ is independent of $x$. In all of the cases we consider here, the relaxation rate of ground state atomic polarization $\gamma$ does not decrease as a function of $x$ faster than $x^{-2}: \gamma$ is independent of $x$ for trapped and cooled atoms; in buffer-gas-free cells, the transit rate $\gamma_{t}$ of atoms through the light beam (which can be the dominant contribution to $\gamma$ ) scales as $x^{-1}$; for cells filled with buffer gas in the diffusion-limited regime, as discussed below, $\gamma_{t}$ scales as $x^{-2}$. Thus for a given light power, $\kappa$ is either independent of $x$ or is improved by decreasing $x$.

[31] N. Davidson, H. J. Lee, C. S. Adams, M. Kasevich, and S. Chu, Phys. Rev. Lett. 74, 1311 (1995).

[32] F. A. Franz and C. Volk, Phys. Rev. A 14, 1711 (1976).

[33] W. Demtröder, Laser Spectroscopy (Springer-Verlag, Berlin, 1998).

[34] H. G. Robinson, E. S. Ensberg, and H. G. Dehmelt, Bull. Am. Phys. Soc. 3, 9 (1958).

[35] M. A. Bouchiat and J. Brossel, Phys. Rev. 147, 41 (1966).

[36] E. B. Alexandrov and V. A. Bonch-Bruevich, Opt. Eng. 31, 711 (1992).

[37] D. Budker, V. Yashchuk, and M. Zolotorev, Phys. Rev. Lett. 81, 5788 (1998).

[38] D. Budker, D. F. Kimball, S. M. Rochester, V. V. Yashchuk, and M. Zolotorev, Phys. Rev. A 62, 043403 (2000).

[39] D. Budker, D. F. Kimball, V. V. Yashchuk, and M. Zolotorev, Phys. Rev. A 65, 055403 (2002).

[40] This light intensity is 100 times larger than the highest intensity at which our calculation has been checked against experiment, but we know of no reason why the calculation should fail under these conditions.

[41] A. D. Wilson-Gordon and H. Friedmann, Opt. Commun. 94, 238 (1992).

[42] V. Wong, R. W. Boyd, C. R. Stroud, Jr., R. S. Bennink, D. L. Aronstein, and Q.-H. Park, Phys. Rev. A 65, 013810 (2001).

[43] I. Novikova, A. B. Matsko, and G. R. Welch (unpublished).

[44] J. Hald, J. L. Sørensen, C. Schori, and E. S. Polzik, Phys. Rev. Lett. 83, 1319 (1999).

[45] A. Kuzmich, L. Mandel, and N. P. Bigelow, Phys. Rev. Lett. 85, 1594 (2000).

[46] D. V. Kupriyanov and I. M. Sokolov, Quantum Opt. 4, 55 (1992).

[47] V. A. Sautenkov, M. D. Lukin, C. J. Bednar, I. Novikova, E. Mikhailov, M. Fleischhauer, V. L. Velichansky, G. R. Welch, and M. O. Scully, Phys. Rev. A 62, 023810 (2000).

[48] L. R. Hunter, Science 252, 73 (1991).

[49] V. Yashchuk, D. Budker, and M. Zolotorev, in Trapped Charged Particles and Fundamental Physics, edited by D. H. E. Dubin and D. Schneider, AIP Conf. Proc. No. 457 (AIP, 
New York, 1999), pp. 177-181.

[50] D. F. Kimball, D. Budker, D. S. English, C.-H. Li, A.-T. Nguyen, S. M. Rochester, A. Sushkov, V. V. Yashchuk, and M.
Zolotorev, in Art and Symmetry in Experimental Physics, edited by D. Budker, P. H. Bucksbaum, and S. J. Freedman, AIP Conf. Proc. No. 596 (AIP, New York, 2001), pp. 84-107. 